\title{
Cayendo como moscas: representaciones mediáticas y sociodigitales de la responsabilidad social universitaria en tiempos de Covid-19
}

\author{
Hugo Méndez Fierros
}

\begin{abstract}
Resumen
Se interpretan las representaciones mediáticas y sociodigitales de las acciones de Responsabilidad Social Universitaria (RSU) de una institución de educación superior pública mexicana frente a la COVID-19. Se analizaron 128 productos periodísticos, 68 publicaciones de Facebook institucional y 13,755 comentarios de la comunidad virtual, en el periodo del 11 de marzo al 11 de mayo del 2020. Los recursos teóricos de las representaciones sociales, de la producción social de la comunicación y agenda setting, empleando como método el análisis de contenido, permitieron confirmar la centralidad de la comunicación en la gestión de un problema mundial en la escala regional. Asimismo, se concluye que durante un momento de crisis, la RSU es fuente de acciones con impactos sociales positivos que retroalimentan recursivamente los vínculos entre identidad regional e identidad universitaria, a través de producciones simbólicas. De las representaciones de la RSU en medio de la pandemia, emergió una categoría analítica denominada "orgullo universitario"; emana como una emoción colectiva enlazada al sentido de pertenencia y a la identidad universitaria. La investigación sobre comunicación y emociones en los entornos mediático-digitales se abre como una veta, si no nueva, que exige ampliar su estudio desde perspectivas interdisciplinarias.

\section{Abstract}

The media and socio-digital representations of the actions of University Social Responsibility (USR) of a mexican public education institution compared to covid-19 are interpreted. 128 journalistic products, 68 publications on the institutional Facebook and 13,755 comments from the virtual community were analyzed, in the period from March 11 to May 11, 2020. The theoretical resources of the Social Representations, of the Social Production of the Communication and Agenda setting, using content analysis as a method, allowed us to confirm the centrality of communication in managing a global problem on a regional scale. Likewise, it is concluded that during a moment of crisis, the RSU is a source of actions with positive social impacts that recursively feedback the links between regional identity and university identity, through symbolic productions. From the representations of the RSU in the midst of the pandemic, an analytical category called University Pride emerged, emanating as a collective emotion linked to the sense of belonging and university identity. Research on communication and emotions in digital-media environments opens up as a vein, if not a new one, which requires expanding your study from interdisciplinary perspectives.

Palabras Clave

Representaciones mediáticas, Representaciones sociodigitales, Responsabilidad social universitaria, Covid-19 Keywords

Media representations, Socio-digital representations, University social responsibility, Covid-19
\end{abstract}

\section{Introducción}

El 2020 quedará marcado en la historia de la humanidad debido a la pandemia generada por la COVID-19. La centralidad de la comunicación en medio de este periodo especial, ha sido incuestionable. En este contexto, el 27 de marzo desde el confinamiento hogareño me comuniqué 
vía WhatsApp al teléfono +41 2250176 90, registrado en Ginebra, Suiza. El bot oficial que la Organización Mundial de la Salud (OMS) había lanzado ese mismo día, respondió con un menú informativo variado.

El contenido emitido por la OMS era compartido entre decenas de miles de ciudadanos en el mundo, comentado por distintos lídredes de Estados nacionales y replicado por medios de comunicación de todo el planeta. Esta forma de gestionar a través de redes sociodigitales la comunicación oficial sobre la emergencia vírica, además de permitirme seguir las últimas noticias me generó un interés académico. Pude distanciarme sanamente del fenómeno y establecer una perspectiva de análisis comunicacional acerca de una cuarentena que era a la vez vivida, narrada e interpretada. Me propuse el objetivo de identificar e interpretar las representaciones que, en torno a las acciones de Responsabilidad Social Universitaria (RSU) de la Universidad Autónoma de Baja California (UABC) frente a la pandemia, construyeron medios regionales (televisión y periódicos). Así como interpretar el contenido de las interacciones en el espacio de Facebook UABC Oficial, con el fin de conocer las representaciones construidas por las comunidades virtuales sobre el mismo tema, durante el periodo del 11 de marzo al 11 de mayo del 2020.

El artículo, está estructurado de la siguiente manera: en el primer apartado, Pensar la Pandemia desde lo simbólico, se revisan los conceptos teóricos básicos de la teoría de las representaciones sociales, de la teoría de la producción social de la comunicación y de la teoría de la agenda setting. Después, se presentan tres subapartados de orden contextual, el primero de ellos, Emergencia sanitaria en un mundo globalizado, trata el surgimiento y propagación mundial del nuevo coronavirus; el segundo, denominado México: construcción simbólica de la Covid-19, trata el arribo de esta enfermedad y la forma en que la presidencia de la República construyó discursivamente y atendió en la práctica este suceso. El sub-apartado complementario es Cayendo como moscas, en el que se describen decisiones y actuación del gobierno del Estado de Baja California.

Posteriormente, en Responsabilidad social universitaria en tiempos de Covid-19, se bosqueja una mirada sobre el rol de las universidades públicas estatales en sus regiones y se define conceptualmente la RSU. Después, en Representaciones mediáticas y sociodigitales, se describe la ruta metodológica seguida y se realiza la discusión de los hallazgos más relevantes, a la luz de los recursos teórico-conceptuales. A manera de cierre, en Consideraciones finales, se esbozan algunas notas para orientar el trabajo académico, hacia la comprensión de fenómenos comunicacionales vinculados a los procesos de construcción de las identidades universitarias y las identidades regionales, en los entornos digitales.

\section{Pensar la pandemia desde lo simbólico}

En este apartado se presenta un corpus integrado por perspectivas teóricas de distinto alcance, para hacer una aproximación a un fenómeno que integra procesos de producción social de comunicación pública y construcción de representaciones en redes sociodigitales. La pandemia por COVID-19 generó transformaciones sociales en gran parte del mundo y los medios de comunicación ofrecieron reelaboraciones de significados para contribuir a efectuar los ajustes necesarios entre lo que cambia en el entorno y lo que conoce acerca de ello la sociedad.

Habrá que partir del concepto de representaciones colectivas, planteado por Émile Durkheim. Las representaciones son realidades de la vida colectiva $y$ mental del sujeto, que pueden influir directamente sobre otras, o bien combinarse. Tanto las representaciones de orden individual como las construidas socialmente se entrelazan en el proceso de reproducción social. Las representaciones "son fenómenos reales dotados de propiedades específicas y que se comparten de forma diferente unos con otras según tengan o no propiedades comunes" (Durkheim, 2006, p. 15). Serge Moscovici, desde la psicología social, arguye que toda representación está compuesta por figuras y expresiones socializadas, una organización de imágenes y lenguaje, los cuales simbolizan actos y situaciones que se convierten en comunes (Moscovici, 1979). La representación comunica y produce comportamientos, que determinan la naturaleza de los estímulos que nos rodean y el sentido de las respuestas que deben darles. Asimismo, el proceso que posibilita otorgar sentido a las representaciones está influenciado por las relaciones entre grupos y personas, resultado de relaciones dinámicas y caracterizado por la producción de comportamientos y vínculos con el medio físico en términos de acción. 
Denise Jodelet, propone que ante la necesidad de querer comprender el mundo que nos rodea, se fabrican representaciones para conocer los objetos, a las personas, las situaciones o las ideas (Jodelet, 1997). Una representación social "designa una forma de conocimiento específico, cuyos contenidos manifiestan la operación de procesos generativos y funcionales socialmente caracterizados, los cuales designan una forma de pensamiento social" (Jodelet, 1984, p. 474). Las representaciones constituyen modalidades cognitivas que se orientan hacia la comunicación, la comprensión y el dominio del entorno social, material e ideal. Dentro de la psicología social, el concepto de representaciones sociales se ha planteado bajo la siguiente acepción: "es una forma de conocimiento específico, el saber del sentido común, cuyos contenidos manifiestan la operación de procesos generativos, y funcionales socialmente caracterizados. En sentido más amplio, designa una forma de pensamiento social" (Jodelet, citada en Moscovici, 1988).

Desde la perspectiva de la producción social de comunicación, se observa que en el mundo contemporáneo la estructura social y el pensamiento individual son elementos que participan entrelazados en la producción de los procesos de comunicación pública, tanto en los medios conocidos como tradicionales (televisión, radio y medios impresos) como en los medios de comunicación digitales. Manuel Martín Serrano, expone que:

Los datos de referencia procedentes de los materiales comunicativos son el producto de una elaboración institucionalizada de la informción previa a la elaboración cognitiva de la información con la que se constituyen las representaciones subjetivas. Es previo que otro Actor (Emisor) distinto del Sujeto cognoscente adquiera algún conocimiento a propósito de algo de lo que ocurre en el entorno y que en el marco de una organziación comunicativa se elabore y se difunda un producto comunicativo, que proporcionará determinada información sobre el objeto de referencia. Solo más tarde el Actor cognoscente (Receptor) tomará en cuenta esos datos, en todo o en parte, para generar o alterar una representación personal de la realidad en la cual puede tener cabida alguna apreciación existente en el relato respecto al acontecer a propósito del que se inició el proceso comunicativo. (Martín, 2017, p.136)
La relevancia de los actores que concurren en la producción institucionalizada de representaciones mediáticas, reside en su papel de mediadores en el proceso de la construcción social de la realidad. La conformación de las corrientes de opinión y la generación de representaciones acerca de los distintos fenómenos del entorno en las sociedades contemporáneas, pasa obligadamente por los medios de comunicación y las redes sociodigitales. Los agentes elaboran representaciones del mundo cimentadas en creencias, principios y valores; esos preceptos otorgan significado a su presente.

En los imaginarios colectivos se van concomitando los elementos de cambio del entorno social, material o ideal para asumir toda novedad. En cada época y en cada sociedad, incorporar cognoscitivamente lo que irrumpe en la realidad o comprender lo que desaparece es una tarea institucional encaminada al control social (Velarde y Bernete, 2016). Las representaciones del mundo, como las prácticas sociales de las personas, son el resultado de conflictos subjetivos y objetivos, individuales y colectivos, que se suscitan entre las necesidades y los valores, entre los deseos y las limitaciones. La comunicación institucional con otras fuentes de información no menos influyentes, puede activar esos conflictos; también puede esforzarse en canalizar el desenlace hacia un estado de conciencia o hacia una actuación inducidos por el Relator. Pero el Relator no puede asegurar con certeza cuál va a ser el resultado que su intervención comunicativa va a provocar sobre la conciencia o sobre los comportamientos de los Destinatarios... [a final de cuentas] La respuesta del sujeto a los acontecimientos no depende tanto de la vía por la que le llega la información, como de las representaciones que elabora con toda información que procese cognitivamente, cuando considere cuál es su implicación en lo que acontece. (Martín, 2017, pp.139-140)

En resumen, las instituciones comunicativas generan y difunden información que consumen y de ella se apropian culturalmente los usuarios. Esta información, regularmente, redunda en el mismo tipo de mediación cognitiva. "Cuando en las redes numerosas personas que pertenecen a la población en su conjunto participan en un tema de debate [como ocurre en la pandemia por la COVID-19] se multiplica la redundancia y la estereotipia, y predominan las representaciones alineadas con 'el orden establecido"” (Martín, p.17, 2019). 
De manera complementaria, en un nivel teórico más operativo, conviene anotar para los intereses de este trabajo, el planteamiento de la teoría de la agenda setting que trata sobre lo que se piensa, pero se refiere también a las opiniones y emociones expresadas sobre determinados temas. La teoría no se limita a la enumeración de una lista de tópicos sino que, en un segundo nivel, la agenda incluye imágenes y perspectivas. Esta dimensión (segundo nivel de agenda) tiene que ver con el modo en que se produce la transferencia de la prominencia sobre un tema (cómo pensar acerca de); no sólo la prominencia de los asuntos, sino también la de los aspectos de esos temas (McCombs, 1996). Esta teoría plantea que no solamente existe la agenda mediática, sino que hay dos; una agenda pública constituida por los tramas de interés para los públicos o audiencias y una agenda política que está integrada por las acciones de respuesta que ofrecen los grupos políticos y las instituciones sociales a distintos temas de interés.

En conjunción con los efectos de tradición en la investigación de la comunicación de masas, los estudios iniciales para el establecimiento de un agenda-setting indagaron en el impacto de el agenda-setting informativo sobre el agenda-setting público. Más recientemente el agenda-setting informativo se ha desplazado de su variable independiente hacia una variable dependiente. La cuestión central de investigación que anteriormente se preguntaba quién establecía el agenda-setting público ha cambiado para preguntarse ahora quién establece el agenda-setting informativo. (McCombs, 1996, p.31) McCombs (1996) concluye que la respuesta a la interrogante anterior es que la agenda-setting de los medios de comunicación se establece de forma compartida, pero que definitivamente los medios y las redes sociales digitales, ejercen una influencia dominante en la conformación de las distintas agendas.

Para cerrar este apratado, es importante destacar que la producción de comunicación institucionalizada en su faceta periodística digital ha vivido en las últimas décadas transformaciones muy importantes, entre ellas, dos que son relevantes para el tema que aquí se analiza: el giro emocional en el periodismo y el nuevo rol activo de los usuarios. Este "giro emocional"se ha originado por los rápidos cambios tecnológicos de la era digital, que han dado paso a un mayor papel de la emoción en la producción noticiosa y en la forma como responden las audiencias a los productos comunicativos
(Wahl-Jorgensen, 2019). Los usuarios de medios y redes digitales, al participar comentando una noticia, demuestran mayor interés en el contenido. El grado en que el formato de la historia, en términos de características multimedia, involucra al usuario con la historia, también puede afectar si se elige o no participar (Ksiazek, 2018).

\section{Emergencia sanitaria en un mundo globalizado}

La contingencia sanitaria que modificó de manera disruptiva la vida social del mundo en el 2020, fue originada por la propagación a escala global de un nuevo coronavirus que causó la COVID-19, una enfermedad infecciosa desconocida hasta antes de que estallara el brote en Wuhan, China, en diciembre de 2019 (OMS, 2020).

El coronavirus no es una enfermedad infecciosa cualquiera. Es una pandemia vírica. La palabra pandemia viene del griego antiguo, y significa «todo el pueblo». En efecto, todo el pueblo, todos los seres humanos, estamos afectados por igual. Pero precisamente eso es lo que no hemos entendido si creemos que tiene algún sentido encerrar a la gente dentro de unas fronteras. (Markus, p. 130, 2020)

El 4 de enero de 2020 la OMS publicó en Twitter un hilo breve acerca de la existencia de un conglomerado de casos de neumonía en China. Esta fue la primera comunicación pública a nivel internacional. Días después, este organismo multilateral alojó un espacio informativo en su página web, con orientaciones sobre el modo de detectar y gestionar posibles situaciones de riesgo. Finalmente, el 11 de marzo quedó inscrito en la historia como la fecha de declaración de existencia de una pandemia por COVID-19 (OMS, 2020).

Para Badiou (2020) el problema de la epidemia fue mal definido y enfrentado desde el principio, porque no atendió la complejidad de superposiciones y entrecruzamientos de factores condicionantes naturales y determinaciones sociales. Byung-Chul Han, escribió que el pánico colectivo se dio como una reacción inmunitaria social, trascendiendo distintas escalas (Han, p. 108, 2020). Ante este pánico, el 13 de marzo fue establecido el Fondeo de Respuesta Solidaria contra la COVID-19 a nivel mundial. La solidaridad y cooperación adquirieron nuevos significados. 
[Ante esta] amenaza universal [se] origina una solidaridad global, nuestras mezquinas diferencias se vuelven insignificantes, todos trabajamos juntos para encontrar una solución: y ahí estamos ahora, solo que en la vida real[...] Lo importante es reflexionar sobre el triste hecho de que necesitamos una catástrofe para ser capaces de repensar las mismísimas características básicas de la sociedad en la que vivimos. (Zizek, 2020, capítulo 4, posición 298)

\section{México: construcción simbólica de la Covid-19}

En los últimos días de febrero del 2020, el presidente Andrés Manuel López Obrador declaró que México estaba preparado para enfrentar una pandemia que a nivel mundial mantenía en alerta máxima a la OMS. Comparó a la COVID-19 con la influenza y arguyó que los actores políticos que se manifestaban contra la estrategia de comunicación y gestión de la crisis sanitaria, eran adversarios con otros intereses (López, 27 de febrero de 2020). En plataformas como Twitter y Facebook, el tema de la actuación del gobierno frente a la pandemia se polarizó rápidamente, como sucede con muchos temas de la agenda política en México. En el marco de la respuesta del Estado, se situó la comunicación al centro, el presidente instituyó conferencias vespertinas diariamente, el vocero designado fue Hugo López-Gatell (López, 28 de febrero de 2020), quien por la exposición mediática se convirtió en una figura pública dentro de México. El segundo mes del año dejó confirmados oficialmente los primeros tres casos positivos de COVID-19 en México. También en ese periodo quedaron establecidas las líneas discursivas centradas en el distanciamiento social, que apelaban a la contención y mantenimiento de ánimo positivo entre la población (SSGM, 28 de febrero de 2020). Fue una estrategia que encontró fuerte oposición en redes sociales y en algunos medios de comunicación, sobre todo con el desgaste que trajo el paso de las semanas.

Las giras de trabajo y las reuniones públicas del presidente continuaron; en la arena mediática este y el uso obligatorio, o no, de cubrebocas, fueron dos temas de debate en la agenda pública. El lunes 23 de marzo inició la Jornada Nacional de Sana Distancia, que se mantuvo vigente hasta el 30 de abril y posteriormente fue extendida hasta el 30 de mayo. Durante este periodo se cancelaron actividades productivas no esenciales, eventos masivos y se suspendieron clases presenciales en todos los niveles educativos (SSGM, 24 de marzo de 2020). Al 30 de mayo, la Secretaría de Salud del Gobierno Federal (SSGM) había registrado 87,512 casos positivos y 9,779 muertes en México (SSGM, 30 de mayo de 2020).

\section{Cayendo como moscas}

México y Estados Unidos de América (EUA) comparten una frontera de 3,200 km de longitud. De esta extensión territorial, la frontera noroeste se encuentra definida a partir de la vecindad entre California y Baja California. De acuerdo con estimaciones del Consejo de Planeación y Desarrollo (COPLADE), la población de Baja California alcanzó la cifra de 3,633,772 habitantes en 2019 y se divide en seis municipios: Ensenada, Mexicali, Playas de Rosarito, San Quintin, Tecate y Tijuana. La capital del estado es Mexicali, que cuenta con una población de 1,065,882 habitantes. No obstante, la mayor localidad urbana es Tijuana, que tiene 1,798,741 pobladores (COPLADE, 2018). En Baja California gobernó por primera vez en la historia de México, un ente político distinto al Partido Revolucionario Institucional (PRI). En 1989 el Partido Acción Nacional (PAN) asumió el poder y lo mantuvo durante 30 años. En 2019, en un momento de grandes cambios políticos, asumió la gubernatura, Jaime Bonilla Valdez, emanado del partido Movimiento de Regeneración Nacional (MORENA) y a su gobierno le ha tocado enfrentar esta pandemia.

El 17 de marzo, fueron confirmados los primeros dos casos de COVID-19 en Baja California, ambos en la ciudad de Mexicali (GEBC, 17 de marzo de 2020). En el mismo tenor de la estrategia gubernamental de comunicación a nivel federal, en esta entidad fronteriza se implantaron transmisiones a través de medios y redes sociales en las cuales se publicaban diariamente datos de casos positivos y decesos; también se habilitó un portal informativo con recomendaciones y avisos. La línea discursiva era luchar unidos contra la COVID-19 y estar alerta contra las acciones desinformadoras y de propagación de fakenews de los adversarios políticos.

El último día de marzo se informó oficialmente sobre la primera muerte por COVID-19 en Baja California (GEBC, 31 de marzo de 2020). Un tema álgido en la agenda pública fue la negativa del grupo de empresas maquiladoras para 
suspender sus actividades catalogadas como no esenciales. El gobernador Jaime Bonilla, difundió su postura de no apoyar a empresas que fabrican ventiladores respiratorios y se negaron a vender sus productos al sector salud de la entidad, que en ese momento estaba rebasado en sus capacidades hospitalarias. Ese mismo día, Baja California se posicionó como la tercera entidad de México con más casos COVID-19 y la segunda en tasa de mortalidad por contagio (GEBC, 10 de abril de 2020). La condición de frontera con EUA fue señalada por las autoridades estatales como el principal factor de crecimiento de contagios y muertes. Los hospitales de Baja California no contaban con suficientes respiradores ni equipamiento médico; por el incremento de contagios entre el personal de las áreas de salud, faltaban enfermeras y médicos (Rosagel, 5 de abril de 2020; Reyes, 12 de abril de 2020). Los resultados de las pruebas enviadas a Ciudad de México tardaban hasta 10 días en llegar. La situación del sistema de salud contribuyó al deterioro de las relaciones políticas entre autoridades estatales y representantes de organismos federales, de lo cual quedó constancia en las narrativas mediáticas (Berriózabal y Gómez, 30 de abril de 2020). El 12 de abril, a través de redes sociales, enfermeras y médicos denunciaron de manera anónima esas carencias en la clínica 20 del IMSS en Tijuana, el tema fue trending topic al circular un video vía Twitter, en el que un actor mexicano leyó una carta enviada por un médico que clamaba ayuda (Gómez, 12 de abril de 2020; Villalobos, 13 de abril de 2020).

El 13 de abril el gobernador de Baja California, hizo público su encono contra las autoridades federales del Instituto Mexicano del Seguro Social (IMSS) durante la conferencia diaria transmitida en Facebook Live; lo hizo metafóricamente y su mensaje se viralizó: "Los médicos están cayendo como moscas [... el el problema del IMSS afectó la salud en el estado, por lo menos aquí en Baja California [...] no se ha puesto las pilas" (El Universal, 13 de abril 2020). Las disputas en redes sociales crecieron, como los casos positivos y las defunciones. Al 30 de mayo Baja California era uno de los estados con mayor incidencia de coronavirus: 138.3 casos por cada 100 mil habitantes. La entidad registraba 864 muertes y 5 mil 027 casos confirmados por COVID-19 (GEBC, 30 de mayo de 2020).

Lo planteado hasta aquí, explica el contexto de actuación de las instituciones de educación superior públicas de Baja
California, en el marco de su responsabilidad social. Durante la crisis sanitaria la RSU adquirió nuevas dimensiones, las universidades tuvieron que innovar y adaptarse de manera ágil. Con un gobierno estatal rebasado en sus capacidades de salud pública, en pugna con autoridades federales y en un espacio transfronterizo que imponía retos colosales por el flujo social imparable, aún con el paso restringido a las actividades esenciales en la frontera, desde el 20 de marzo por los gobiernos de México y EUA (Secretaría de Relaciones Exteriores de México, 2020). Las universidades públicas debían cumplir sus funciones sustantivas con mayor impacto social, por su papel histórico en el desarrollo regional. En términos mediáticos la actuación de la UABC fue especialmente observada y representada, de acuerdo con los resultados que se exponen más adelante.

\section{La Responsabilidad Social Universitaria en tiempos de Covid-19}

La universidad como institución ocupa un papel relevante en la vida de las sociedades contemporáneas. Con siglos de tradición a cuestas, se ha movido en oleajes continuos entre rasgos estructurales que permanecen casi inmutables y fuertes tormentas innovadoras. Por ello, resulta de interés observar la actuación de estas instituciones frente a la pandemia por la COVID-19. En México se dieron dos eventos en el campo de la educación superior que marcaron un cambio en la inercia de la gestión del riesgo por el nuevo coronavirus. Las dos instituciones líderes en el ámbito privado y público, Tecnológico de Monterrey y la Universidad Nacional Autónoma de México (UNAM), aceleraron las medidas de confinamiento. Por un lado, el Tecnológico de Monterrey anunció el 12 de marzo la cancelación de clases presenciales en todos sus niveles y el inicio de sus programas virtuales de continuidad académica en línea. Se anticipó a la suspensión de actividades oficial del gobierno federal (Tecnológico de Monterrey, 2020).

Por otra parte, la UNAM informó el mismo día que fortalecería acciones preventivas y para ello, se cancelarían eventos masivos en espacios cerrados e iniciaría con la preparación de un proyecto académico para que estudiantes y profesores continuaran sus trabajos desde casa (UNAM, 2020). Tres días después, se efectuó una reunión entre el secretario de Educación Pública Federal, Esteban Moctezuma Barragán, 
y los secretarios de educación de las 32 entidades federativas de México, ahí tomaron el Acuerdo 02/03/20, publicado en el Diario Oficial de la la Federación, para suspender clases en todos los niveles del sistema educativo nacional del 23 de marzo al 17 de abril (Secretaría de Educación Pública, 2020). Este fue el inicio del confinamiento social para amplios grupos de la población vinculados al sector educativo. El 17 de marzo la Asociación Nacional de Universidades e Instituciones de Educación Superior (ANUIES) convocó a los rectores y rectoras de las 197 instituciones públicas y privadas agremiadas, para reunirse con el Subsecretario de Educación Superior, Luciano Concheiro Bórquez; en la sesión acordaron que el último día de clases fuera el 20 de marzo y se programó el retorno para el 20 de abril, posteriormente, se extendió el regreso hasta el siguiente ciclo escolar (ANUIES, 2020).

La circunstancia de la educación escolarizada en medio de la pandemia es singular, pues, no hay registro de otro momento de la historia en que se hubiesen suspendido las actividades de más de 1,215 millones de estudiantes, de todos los niveles educativos a escala mundial. Derivado de lo anterior, en las páginas web y las redes sociodigitales institucionales de cientos de universidades en México y el mundo, se percibió un concierto académico en torno a la crisis por la COVID-19. Especialistas de todas las áreas científicas y humanísticas coinciden en el estudio de los múltiples factores que acompañan el surgimiento del nuevo coronavirus (Casanova, 2020). En este escenario, las universidades públicas estatales como organismos descentralizados que funcionan con recursos provenientes de la federación y de los gobiernos de las entidades en las que se encuentran establecidas, fueron llamadas a demostrar su RSU a las comunidades en donde están enclavadas. En general, se puede advertir que son universidades de profunda raigambre, en la mayoría de los casos documentados, han construido un prestigio social derivado de sus contribuciones al desarrollo de sus regiones. Actualmente, existen 34 universidades de este tipo en México, una por cada entidad, con excepción de la Ciudad de México; asimismo, habrá que anotar que las entidades de Chihuahua, Campeche y Sonora, cuentan con dos de ellas (Integralia, 2019). Estas universidades también juegan un rol importante en la construcción de las identidades regionales; ahí, la responsabilidad social, por ser una acción de impacto social positivo, es una expresión que retroalimenta el sentido de pertenencia al territorio cultural. Al analizar distintos discursos históricos, se constató que la misión atribuida a las IES conlleva el ser factores decisivos para el impulso del desarrollo y la solución de las problemáticas de la entidad regional a la que pertenecen (Piñera, 2008).

En Baja California, la UABC ha sido un factor clave del desarrollo económico, social, cultural y político. Fundada en 1957, actualmente atiende una matrícula de más de 65,500 estudiantes en 134 programas educativos de licenciatura y 65 de posgrado. Integra 41 facultades, escuelas e institutos distribuidos en tres campus ubicados en Ensenada, Mexicali y Tijuana; además, mantiene presencia en zonas suburbanas y rurales de todo el estado. En Baja California seis de cada diez alumnos del nivel superior estudian en la UABC (UABC, 2020).

La UABC cuenta con 531 académicos miembros del Sistema Nacional de Investigadores (SNI) que trabajan en 626 proyectos de investigación y están agrupados en 227 cuerpos académicos. Su vinculación con los diversos sectores le permite colaborar en 589 convenios y actualmente más de 19,600 estudiantes prestan servicio social comunitario. En el ámbito internacional, mantiene acuerdos con 392 universidades de distintas partes del mundo, de las cuales recibe anualmente profesores y estudiantes visitantes; en el primer semestre del 2020 recibió a 481 alumnos de otras IES (UABC, 2020).

La RSU es uno de los tres ejes transversales de su Plan de Desarrollo Institucional (PDI) 2019-2023. Ahí declara que la RSU representa un compromiso institucional autónomo, colectivo, en el que concurren personas e instituciones, en la orientación del cumplimiento de su misión hacia la pertinencia social con transparencia y rendición de cuentas, frente a los retos de equidad, inclusión y a los desafíos ambientales locales y globales (UABC, 2019). Finalmente, establece que para desarrollar la RSU se requiere producir conocimientos que contribuyan a revertir la desigualdad e impacten en la satisfacción de las necesidades del entorno. No obstante, la generación de estos conocimientos debe favorecer la democratización e innovación con inclusión social (UABC, 2019).

Para la ANUIES, la noción de responsabilidad social debe orientar a las universidades hacia el impulso de procesos 
de transformación al interior de sus comunidades para promover una formación humanística, crítica y reflexiva que revalore los sentidos de la paz, el progreso, la justicia social y los derechos humanos; divulgar la ciencia y la cultura bajo el principio de libertad y compromiso social; proponer soluciones innovadoras ante los problemas científicos, políticos, económicos y sociales de México; impulsar la investigación pertinente que conytribuya a la solución de las problemáticas locales y regionales; y promover la inclusión y la equidad de oportunidades en la educación superior (ANUIES, 2012). La clave de una correcta RSU es definirla, operarla y evaluarla, en función de sus impactos en todos sus ámbitos de gestión, formación, cognición y participación social; orientarla vigoriosamente hacia el desarrollo sostenible de la sociedad, y convocar transversalmente a toda la comunidad universitaria para implementar políticas y programas que transformen a la universidad, partiendo de autodiagnóstico desarrollado con coherencia, transparencia y ánimo de innovación (Vallaeys, 2018).

La RSU: Es una política de gestión de la calidad ética de la universidad que busca alinear sus cuatro procesos (gestión, docencia, investigación y extensión) con la misión universitaria, sus valores y compromiso social, mediante el logro de la congruencia institucional, la transparencia y la participación dialógica de toda la comunidad universitaria (autoridades, estudiantes, docentes, administrativos) con los múltiples actores sociales interesados en el buen desempeño universitario y necesitados de él, para la transformación efectiva de la sociedad hacia la solución de sus problemas de exclusión, inequidad y sostenibilidad. (Vallaeys, 2006, p. 5)

Finalmente, La RSU debiera ser per sé una finalidad transversal a las IES, sin someterse a evaluaciones que condicionen, en las universidades públicas, su financiación, $\mathrm{y}$ en el sector de las universidades privadas, una instrumentalización de su fin social. Sin embargo, el creciente número de instituciones privadas frente a las públicas condiciona la importancia de establecer una redefinición de cuáles son las responsabilidades que las IES deben cumplir para que la educación superior suponga un bien público y no se limite a un servicio condicionado por oferta y demanda en un mercado de libre comercio (Martí-Nogera, Calderón
Fernández-Godenzi, 2018). Una vez planteado el problema, el marco teórico conceptual y el contexto del objeto de estudio, en el siguiente apartado se presenta el método y la discusión de los resultados.

\section{Representaciones mediáticas y sociodigitales}

El marco epistemológico en el que se inscribe este trabajo de investigación es el interpretativo, que privilegia la búsqueda de significados en los discursos sociales e interpreta la realidad empírica en los términos comprendidos por los actores sociales productores de las narrativas a analizar (Delgado, 2007). Dicha vertiente epistemológica da fundamento a la metodología cualitativa, que se refiere en su más amplio sentido a la investigación que produce datos descriptivos a partir de las palabras habladas o escritas y de la conducta observable de las personas, grupos o instituciones en su intersubjetividad (Taylor Bogdan, 1987).

El método utilizado en la construcción de los datos fue el análisis de contenido, por convenir al objetivo de interpretar las representaciones mediáticas en torno a las acciones de RSU desarrolladas por la UABC en el marco de la pandemia por la COVID-19, así como las representaciones contenidas en los comentarios, expresiones e interacciones discursivas de la comunidad virtual participante en la página de Facebook UABC Oficial (@OficialUabc), sobre el mismo tema, en el periodo del 11 de marzo al 11 de mayo del 2020. En su dimensión técnica el análisis de contenido permite hacer inferencias replicables y válidas de todo tipo de datos. Como herramienta científica contribuye a fortalecer la comprensión de los fenómenos socioculturales; actualmente, más allá de las raíces periodísticas iniciales de la técnica, ha migrado del análisis de contenido a varios campos y a la aclaración de muchos problemas metodológicos (Krippendorff, 2018).

El análisis de contenido es un conjunto de procedimientos interpretativos de productos comunicativos en lenguajes, formatos y soportes tecnológicos variados que derivan de procesos singulares de comunicación previamente registrados. Lo anterior con el uso de técnicas de medida, a veces cuantitativas, a veces cualitativas (Piñuel, 2002). En el análisis de contenido de orden cuantitativo, el proceso empírico es independiente del investigador; en el análisis de mensajes cualitativos, el estudioso está mayormente implicado. No 
obstante, la línea divisoria entre los órdenes cuantitativo y cualitativo del análisis de contenido, es delgada y porosa. En todo caso, es mejor que el enfoque esté centrado en las estrategias analíticas empleadas y los supuestos subyacentes (Neuendorf, 2017).

Los resultados de esta investigación reportan el análisis de 128 productos periodísticos publicados en los espacios digitales de las nueve empresas mediáticas de Baja California con mayor impacto y conveniencia para este estudio; por los factores de circulación en formatos analógicos y digitales, trabajo se enfocó en la fuente educativa y cobertura regional en la frontera noroeste de México y EUA, ver Tabla 1. De igual manera, se analizó el contenido de 68 publicaciones institucionales (posts) en la página de Facebook UABC Oficial y 13,755 comentarios de la comunidad virtual.

\begin{tabular}{|c|c|c|c|c|}
\hline Medio & $\begin{array}{l}\text { Año } \\
\text { de } \\
\text { fundación }\end{array}$ & Página web & Página Facebook & $\begin{array}{l}\text { Seguidores } \\
\text { Facebook } \\
29 / 05 / 2020\end{array}$ \\
\hline $\begin{array}{l}\text { Canal } \\
4 \\
\text { Televisa }\end{array}$ & 1957 & www.televisaregional.com/baja-c & ia@TelevisaMexicaliCanal4 & 395,740 \\
\hline $\begin{array}{l}\text { Canal } \\
66 \\
\text { Intermedi }\end{array}$ & 1997 & www.canal66.tv & @ canal66tv & 524,988 \\
\hline $\begin{array}{l}\text { El } \\
\text { Mexicano }\end{array}$ & 1959 & www.el-mexicano.com.mx & @ElMexicanoOnline & 841,694 \\
\hline $\begin{array}{l}\text { El Sol } \\
\text { de } \\
\text { Tijuana } \\
\end{array}$ & 1989 & elsoldetijuana.com.mx & @ElSoldeTijuana.OEM & 125,395 \\
\hline $\begin{array}{l}\text { El } \\
\text { Vigía }\end{array}$ & 2002 & www.elvigia.net & @elvigia & 169,901 \\
\hline Frontera & 1999 & www.elimparcial.com/tijuana & @ frontera & $1,600,000$ \\
\hline $\begin{array}{l}\text { La } \\
\text { Crónica }\end{array}$ & 1990 & www.elimparcial.com/mexicali & @ lacronicacom & 895,804 \\
\hline $\begin{array}{l}\text { La } \\
\text { Voz } \\
\text { de la } \\
\text { Frontera }\end{array}$ & 1964 & www.lavoz.delafrontera.com.mx & @ lavozdelafrontera & 314,883 \\
\hline Zeta & 1980 & www.zetatijuana.com & @ zeta.tijuana & 246,544 \\
\hline
\end{tabular}

Fuente: Elaboración propia.

Una vez seleccionado el universo de productos comunicativos; con base en la teoría, en los datos empíricos y en la propia experiencia, elaboré las categorías de análisis que se componen de cinco ejes temáticos a saber: a).-Responsabilidad Social Universitaria (RSU): en esta se registraron todas las publicaciones que tratan temas específicos de las acciones desarrolladas por la universidad para impactar positivamente a distintos sectores de la sociedad, en relación con las problemáticas derivadas por la COVID-19; de orden informativo, educativo y de asesoría especializada; de producción de materiales, insumos y equipos; creación de espacios de atención pública; desarrollo de obras de infraestructura y trabajos de orden altruista o filantrópico. b).-Plan de Continuidad Académica (PCA): en esta categoría se consideraron las publicaciones en torno a las estrategias institucionales para la realización de tareas docentes y de investigación durante la suspensión de actividades presenciales; de asesoría en gestión escolar y servicios estudiantiles variados; y de apoyo bibliotecario, entre otras.

c).-Estudiantes Internos de Medicina y Enfermería (EIMF): Aquí se agrupó la información publicada en relación a las actividades de los estudiantes del área de la salud, que realizaban en ese periodo un internado en hospitales públicos como parte de su formación académica obligatoria. d).-Nuevo Ingreso (NI): en esta categoría se registró toda la producción simbólica en torno a los exámenes de nuevo ingreso, trámites y ajustes de fechas para los aspirantes a ingresar a la UABC. Y finalmente, e).-Otros temas (OT): aquí fueron agrupadas las publicaciones relativas a temas variados, como ejemplo: sorteos universitarios y actividades de Fundación UABC. De acuerdo a los objetivos de este artículo y por consideraciones de espacio, se concentraron el análisis y los resultados a presentar, en los textos referidos a la categoría de la RSU. Definidas las categorías, se procedió a codificar la información. Más de la mitad del volumen de la producción periodística se concentró en las acciones de RSU, el $52 \%$ de los registros correspondieron a este tópico. Mientras que el $20 \%$ fue para los temas del PCA y cercano en porcentaje, estuvieron los relativos a EIME con $18 \%$. Finalmente, quedaron las categorías de NI y OT con $5.4 \%$ y $4.6 \%$, respectivamente, como lo ilustra la Tabla 2. 
Tabla 2.

Publicaciones en medios de comunicación, por fechas y categorías temáticas.

\begin{tabular}{|c|c|c|c|c|c|}
\hline Periodo & $\begin{array}{l}\text { Categoría } \\
\text { RSU }\end{array}$ & $\begin{array}{l}\text { Categoría } \\
\text { PCA }\end{array}$ & $\begin{array}{l}\text { Categoría } \\
\text { EIME }\end{array}$ & $\begin{array}{l}\text { Categoría } \\
\text { NI }\end{array}$ & $\begin{array}{l}\text { Categoría } \\
\text { OT }\end{array}$ \\
\hline $\begin{array}{l}11-18 \\
\text { marzo }\end{array}$ & 3 & 10 & 0 & 1 & 0 \\
\hline $\begin{array}{l}19-26 \\
\text { marzo }\end{array}$ & 0 & 1 & 1 & 0 & 0 \\
\hline $\begin{array}{l}27 \\
\text { marzo-3 } \\
\text { abril }\end{array}$ & 15 & 6 & 0 & 4 & 3 \\
\hline $\begin{array}{l}4-11 \\
\text { abril }\end{array}$ & 11 & 0 & 7 & 0 & 0 \\
\hline $\begin{array}{l}12-19 \\
\text { abril }\end{array}$ & 17 & 0 & 12 & 2 & 1 \\
\hline $\begin{array}{l}20-27 \\
\text { abril }\end{array}$ & 9 & 7 & 0 & 0 & 0 \\
\hline $\begin{array}{l}28 \\
\text { abril-5 } \\
\text { mayo }\end{array}$ & 10 & 1 & 2 & 0 & 0 \\
\hline $\begin{array}{l}6-11 \\
\text { mayo }\end{array}$ & 2 & 0 & 1 & 0 & 2 \\
\hline Total & $\begin{array}{l}67 \\
(52 \%)\end{array}$ & $\begin{array}{l}25 \\
(20 \%)\end{array}$ & $\begin{array}{l}23 \\
(18 \%)\end{array}$ & $\begin{array}{l}7 \\
(5.4 \%)\end{array}$ & $\begin{array}{l}6 \\
(4.6 \%)\end{array}$ \\
\hline
\end{tabular}

La presentación por fecha y categoría temática, permite ilustrar la construcción de agenda realizada por los medios de comunicación; partiendo del lanzamiento del plan de continuidad académica, que entre el 11 de marzo y el 3 de abril colocó en la discusión pública el incio de la suspensión de clases y los co-relatos sobre la incertidumbre inicial; por otra parte, se observa una centralización en las acciones de RSU en prácticamente todo el periodo del 11 de marzo al 11 de mayo; el tercer apartado refiere sucesos focalizados entre el 4 y el 19 de abril en relación al tema de los estudiantes internos en hospitales públicos.

Los hallazgos relativos a la producción periodística sobre la RSU de la UABC y la COVID-19, pueden ser analizados a la luz de los planteamientos teóricos de las representaciones sociales, la producción social de la comunicación y la agenda setting, en el sentido de que los cambios en el acontecer, como el vivido con la emergencia sanitaria, generan la necesidad de adaptarse y comprender el mundo que nos rodea, por ello, se fabricaron representaciones para conocer la condición de la pandemia y sus afectaciones a la vida social, llámese "cuarentena", "Jornada de Sana Distancia", "nueva normalidad" o "periodo pos-pandémico"; el sistema de comunicación contribuye a la construcción de estas representaciones orientadas a reducir incertidumbre, a mantener el orden y el control social (Moscovici, 1988; Jodelet, 1997; Martín y Velarde 2015; Martín, 2017; Martín 2019).

En el caso de los resultados obtenidos, los medios de comunicación elaboraron representaciones sobre las acciones de RSU, a partir de tres líneas discursivas centrales: la UABC como casa del saber, genera conocimiento e información que es valiosa para la comunidad y esta tiene el deber de extender estos beneficios a las distintos grupos sociales. Esta universidad ha sido construida simbólicamente como la máxima casa de estudios en Baja California y sus tareas sustantivas generan procesos de impacto social positivo lo que, según Vallaeys, F. (2018, 2006), es el principal atributo de la RSU. La UABC se reposicionó como una institución relevante en la región, a través de acciones espontáneas y voluntarias de grupos de universitarios que produjeron materiales, insumos y equipamientos; así como, por la apertura para brindar servicios de atención pública, en medio de la pandemia y con un gobierno estatal rebasado.

Como señala McCombs (1996), en la actualidad los medios y las redes sociodigitales ejercen una influencia dominante en la conformación de las distintas agendas, los resultados de este análisis así lo ilustran, al dar mayor cobertura los medios regionales a los temas de RSU. La participación de los ususarios que interactuaron en las redes sociales fue consistente con la agenda setting informativa, como se podrá ver más adelante.

Como ejemplos de la producción discursiva en los medios, se muestran los encabezados siguientes: "UABC implementó un plan de atención de contingencia con el fin de mantener informada a la comunidad por medio de un sitio web". "La UABC, en coordinación con la Secretaria de Salud pusieron en marcha el 'Sistema de Telesalud Covid-19'". Y en lo referente a sus tareas sustantivas: "Investigadores de la UABC han estudiado los efectos de propagación del coronavirus". "Para que las pruebas de covid-19 se realicen rápidamente en Baja California, la UABC instaló el Centro de Diagnóstico Covid-19, en el que un grupo de académicos universitarios realizarán pruebas". "La UABC sigue trabajando para llevar la cultura hasta los hogares de la comunidad bajacaliforniana y brindar un momento de esparcimiento, principalmente, a los más pequeños". El impacto de las RSU de la UABC en 
la agenda mediática y en la generación de opinión pública favorable, se puede ejemplificar con estas publicaciones: "Docentes y estudiantes de UABC están trabajando en la creación de mascarillas y caretas de protección, emplean el proceso de impresión 3D". "Estudiantes y maestros de la UABC de las áreas de Ciencias de la Salud e Ingeniería trabajan para combatir el covid-19, con el desarrollo de ventiladores, gel sanitizante y máscaras de protección". "Seis ventiladores respiratorios con un valor de 40 mil pesos, los cuales serán distribuidos a instituciones de salud que lo requieran, fueron elaborados por docentes de la UABC". "Un grupo de universitarios diseñaron una cápsula de traslado médico con inversión negativa, la cual permite filtrar el aire del interior al exterior; protegiendo al personal médico". "Docentes de la UABC, se dieron a la tarea de diseñar y fabricar 20 cámaras de intubación hechas de acrílico y poliestireno, con la finalidad de donarlas al Hospital General y apoyar al personal médico en sus labores en la emergencia sanitaria".

O bien, en la apertura de los espacios universitarios a la sociedad: "Elementos de la Segunda Región Militar improvisaron en las instalaciones de la UABC, un hospital para atender a posibles enfermos de coronavirus". "La Facultad de Medicina de la UABC será un laboratorio certificado para detectar covid-19, de forma gratuita". "Con un déficit en recursos financieros, pero un amplio recurso humano, la UABC instaló Centro de Diagnóstico Covid-19". Y finalmente, "Construye UABC y la IP Hospital Móvil Covid-19. Un hospital con capacidad para 50 camas y dos unidades de Cuidados Intensivos, se construirá en la Facultad de Medicina en Mexicali”. Lo anterior es congruente con lo que apuntan Martín y Velarde (2015), en el sentido de que las representaciones sociales se generan en la comunicación $\mathrm{y}$ en ella se mantienen. Son transmitidas y apropiadas culturalmente, hasta que derivan en concepciones socialmente compartidas que generalmente son aceptadas como patrones de guía en la práctica del mundo de vida. Son las visiones del ser y del no ser, que se pueden denominar con toda pertinencia, representaciones colectivas.

Los resultados que aquí se presentan, también muestran que en el contexto de la pandemia por COVID-19, las emociones son vividas con mayor intensidad y se comunican de diversas formas. Temor, ansiedad, incertidumbre, orgullo, entre otras, fueron proyectadas discursivamente y cobraron cierto impacto en las representaciones. Esto coincide con investigaciones desarrolladas sobre temas relacionados con las emociones y la participación de los usuarios en el periodismo digital; Segado-Boj et al. (2020), señalan que la teoría de la difusión emocional (o emotional broadcaster theory) establece que los individuos tienen la necesidad innata de compartir experiencias, compartir noticias emotivas en redes sociodigitales puede satisfacer esa necesidad. El lenguaje es la forma más común y confiable para que las personas traduzcan sus pensamientos y emociones internas en una forma que otros puedan entendercómo las personas están experimentando el mundo (Tausczik Pennebaker, 2009).

Durante la emergencia sanitaria, el perfil de Facebook (fan page) de la UABC Oficial, se dedicó a informar sobre la COVID-19 y las acciones tomadas por la universidad como respuesta al fenómeno pandémico. Durante el periodo analizado en este artículo, se registró un aumento de 8,619 likes orgánicos que se traducen en un creciente interés de pertenecer a la comunidad virtual de la UABC. En el caso de los mensajes institucionales difundidos en esta red social, la mayor producción de posts caen dentro del eje temático de la RSU, como se puede apreciar en la Tabla 3, lo cual generó a su vez mayor interacción entre las comunidades virtuales respecto a este tópico; en alineación con la producción de relatos periodísticos, como fue anotado anteriormente. 
Tabla 3.
Publicaciones institucionales (posts) en Facebook UABC Oficial, por fechas y categorías temáticas.

\begin{tabular}{llllll}
\hline Periodo & \multicolumn{5}{c}{ Categoría Categoría Categoría Categoría Categoría } \\
& RSU & PCA & EIME & NI & OT \\
\hline $\begin{array}{l}11-18 \\
\text { marzo }\end{array}$ & 2 & 3 & 0 & 0 & 0 \\
\hline $\begin{array}{l}19-26 \\
\text { marzo }\end{array}$ & 6 & 3 & 0 & 0 & 2 \\
\hline $\begin{array}{l}27 \\
\text { marzo-3 }\end{array}$ & 7 & 2 & 0 & 1 & 0 \\
abril & & & & & \\
\hline $\begin{array}{l}4-11 \\
\text { abril }\end{array}$ & 6 & 2 & 1 & 0 & 0 \\
\hline $\begin{array}{l}12-19 \\
\text { abril }\end{array}$ & 9 & 0 & 0 & 0 & 0 \\
\hline $\begin{array}{l}20-27 \\
\text { abril }\end{array}$ & 6 & 1 & 0 & 0 & 1 \\
\hline $\begin{array}{l}28 \\
\text { abril-5 }\end{array}$ & 7 & 0 & 1 & 2 & 0 \\
mayo & & & & & \\
\hline $6-11$ & 3 & 2 & 0 & 0 & 0 \\
mayo & & & & & \\
\hline Total & 46 & 13 & 2 & 3 & 3 \\
\hline & & & & & \\
\hline
\end{tabular}

Lo anterior se ilustra con estos ejemplos de extractos de la producción discursiva en los posts institucionales, que comunicaban y promocionaban el cumplimiento de la RSU, durante la emergencia santitaria por COVID-19, por ejemplo: "Un equipo de docentes de la UABC inició la fabricación de un prototipo de ventilador respiratorio que sea fácil de replicar y con él construir más aparatos". "Se ofrecen servicios bibliotecarios y actividades culturales disponibles en línea para la comunidad cimarrona y sociedad en general". "Agradecemos a todas y todos los alumnos, docentes y egresados de diversas unidades académicas que han estado apoyando con el desarrollo de caretas de protección". "Se compartirá algunas consideraciones para las personas en duelo en tiempos de COVID-19. La transmisión en vivo será a las 8 pm desde la página de Comité de Salud Mental”, entre otros.

Los temas de la RSU se promocionaron y se colocaron en la agenda de la comunidad virtual, sobre ello pensaron, se expresaron y discutieron los actores sociales de estos entornos digitales (McCombs, 1996), como lo ilustra la Tabla 4.
Tabla 4.
Publicaciones institucionales sobre acciones de RSU en Facebook UABC Oficial e interacciones orgánicas.

\begin{tabular}{ll}
\hline Concepto & Cantidad \\
\hline Publicaciones Institucionales (posts) & 46 \\
\hline Comentarios & 2,875 \\
\hline Veces compartidas & 9,840 \\
\hline Clics & 160,178 \\
\hline Visualizaciones & 212,935 \\
\hline Alcance Fuente: Elaboración propia.
\end{tabular}

La producción discursiva en esta red sociodigital relativa a las publicaciones institucionales sobre las acciones de RSU emprendidas por la UABC, se ejemplifica en las siguientes elaboraciones textuales extraídas como muestra de los comentarios de los usuarios, que proyectan cargas emocionales positivas: "Orgullosamente Cimarrones". "Mil gracias, por ayudarnos a protegernos". "Orgullo Cimarrón". "Bravo mil veces ¡Excelente trabajo! ¡Cimarrones!” "Wow excelente jóvenes felicitaciones orgullo cimarrón". "Mucha fortaleza. Bendiciones jóvenes. Preocupados siempre por la comunidad. Gracias por su aportación”. “¡Un gran ejemplo y un gran orgullo muchachos Dios los bendice! Siempre a la vanguardia UABC". "Gracias por su solidaridad". " Sin duda la UABC, presente en la comunidad!" "Cimarrón mi corazón por siempre". Como establece la teoría de las representaciones sociales, se expresan a través del lenguaje las emociones y las perspectivas de la realidad, que forman parte central de las representaciones del mundo, las cuales comunican y producen comportamientos, que determinan la naturaleza de los estímulos que nos rodean y la orientación de nuestras respuestas a ellos (Moscovici, 1979; Jodelet, 1984).

Otras elaboraciones simbólicas que reafirman la emocionalidad -ver Figura1- presente en los integrantes de la comunidad virtual del Facebook UABC Oficial, ante lo que ellos evaluaron como cumplimiento de la RSU, son: "Excelente trabajo colaborativo, hermosa muestra de apoyo y solidaridad". "Qué bonito sacar lo mejor que tenemos en el corazón". "Que orgullo, mi Alma Mater de nuevo se anota un punto más en apoyo a nuestra comunidad". "Te amo UABC, trabajando por tu comunidad eres la mejor”. “EEsa es la actitud! más gestión, más soluciones y menos quejas, felicidades UABC"; como señala Martín Serrano (2015), los medios realizan una función mediadora de ajuste colectivo, a través de la construcción y difusión informativa de la que 
se apropian las audiencias, incorporando esos datos a su cosmovisión.

En el mismo sentido, se puede establecer que el lenguaje es una forma común utilizada para expresar y traducir los pensamientos y emociones internas, de tal forma que usuarios y usuarias de medios digitales no se limitan a recibir pasivamente la información sino que participan en la construcción de narrativas sobre los temas del mundo que habitan (Hermida, 2011; Segado-Boj et al., 2020; Tausczik Pennebaker, 2020).

Figura 1. Nube de palabras de los comentarios vertidos en las publicaciones institucionales sobre acciones de RSU en

Facebook UABC Oficial 11/03/20-11/05/2020.

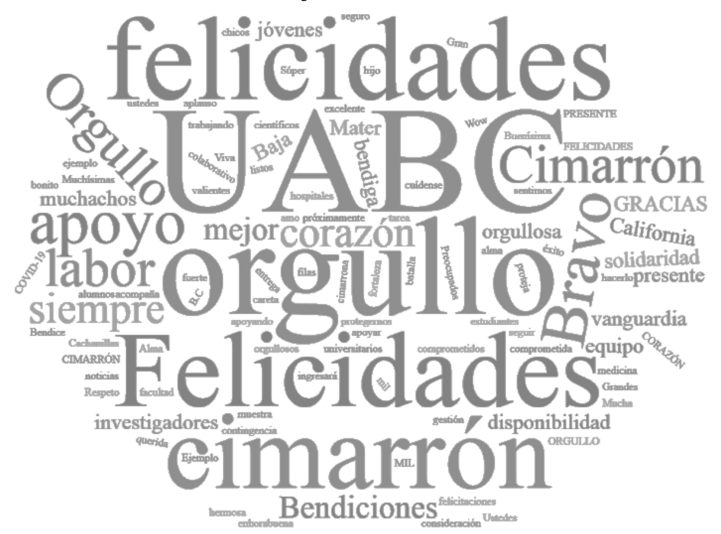

Fuente: Elaboración propia.

Esta nube ilustra las palabras enunciadas con mayores índices de frecuencia, se pueden interpretar como conceptos clave de las representaciones discursivas elaboradas en torno a las acciones de RSU. Es la respuesta de los usuarios de Facebook UABC Oficial ante las representaciones emandas de la cobertura mediática, de los posts de la propia fan page y sobre todo, es una expresión emocional en medio de una pandemia amenzante. Frente a la crisis desencadenada, ha generado respuestas positivas diversas, entre ellas, manifestaciones de orgullo colectivo ligadas a nuevas formas de cooperación. Se ha originado una solidaridad global que permea en regiones y localidades. Ante el difícil escenario de la emergencia vírica hay que trabajar en conjunto para encontrar una solución ante un fenómeno real, que obliga a reflexionar por qué la humanidad requiere de una catástrofe para ser capaz de repensar las características básicas de la sociedad en la que vivimos (Zizek, 2020).

\section{Consideraciones finales}

En este apartado se anotan algunas consideraciones finales, que tienen como propósito esbozar líneas hacia la comprensión de fenómenos comunicacionales vinculados a procesos de construcción de identidades universitarias e identidades regionales, en entornos sociodigitales. Las representaciones de la RSU generadas en los medios de comunicación y en las redes por la comunidad universitaria, se enmarcaron en un momento de cambios sociales, desinformación e incertidumbre, con un gobierno estatal rebasado en algunos renglones de la gestión de esta crisis; lo que permitió a sectores organizados e instituciones diversas, asumir un rol proactivo y ocupar un lugar protagónico en la agenda mediática.

Las representaciones sobre las acciones de RSU de la UABC, se condensan alrededor del cumplimiento de su servicio a la sociedad, innovación y desarrollo de tareas con impacto positivo en las audiencias, lo cual quedó manifiesto en la discursividad elaborada, sobre todo por la comunidad vinculada a la UABC. De lo anterior emergió una categoría que se puede denominar orgullo universitario, que emana como una emoción colectiva enlazada al sentido de pertenencia y a la identidad universitaria.

El orgullo univeritario se puede caracterizar, a priori, como una expresión emocional de la identidad que se experimenta en los rituales institucionales y se comunica a través del lenguaje. La investigación sobre la comunicación y las emociones en los entornos mediáticos digitales no es nueva, pero tampoco ha sido acabada; lo cual exige nuevas aproximaciones desde perspectivas interdisciplinarias.

Asimismo, se reafirmó el supuesto de que las universidades públicas estatales juegan un rol protagónico en la vida social de las regiones donde están asentadas; y se infiere que durante un momento histórico especial como el de una pandemia, es la RSU una fuente de acciones centrales que retroalimentan los vínculos entre la identidad regional y la identidad universitaria. La sociedad espera de las universidades públicas estatales, grandes aportaciones al desarrollo, cuando estas expectativas se cumplen a través de acciones de RSU, el prestigio social y el arraigo regional de las instituciones educativas tiende a fortalecerse. 
Finalmente, quedó manifiesto que tanto en la escala mundial, como en la nacional y la estatal, las acciones para enfrentar a la pandemia generada por la COVID-19, dieron un lugar de centralidad a la comunicación en los planos mediático y de redes sociodigitales. Por ello, el enfoque comunicacional utilizado, el corpus teórico conjuntado, el marco epistemológico interpretativo, una mirada cualitativa y el método del análisis de contenido, funcionaron como una caja de herramientas útil. Habrá que continuar su aplicación y mejorar su efectividad en trabajos venideros.

\section{Referencias}

Asociación Nacional de Universidades e Instituciones de Eduacción Superior (ANUIES) (2012). Inclusión con responsabilidad social: Elementos de diagnóstico y propuestas para una nueva generación de políticas de educación superior. Recuperado de http://www.anuies .mx/noticias/inclusion-con-responsabilidad-social-el ementos-de-diagnostico-y

Asociación Nacional de Universidades e Instituciones de Eduacción Superior (ANUIES) (18 de marzo de 2020). Implementan medidas preventivas con relación al COVID-19 para las instituciones de educación superior en el país [Comunicado de prensa] Recuperado de http://www.anuies.mx/noticias/implementan-med idas-preventivas-con-relacin-al-covid-19-para-las

Badiou, A. (2020). Sobre la situación epidémica. En: P. Amadeo (Ed.), Sopa de Wuhan: Pensamiento contemporáneo en tiempo de pandemias (pp. 67-78). Aislamiento Social Preventivo y Obligatorio. https: //lareverde.files.wordpress.com/2020/04/sopa-de -wuhan-aspo.pdf

Berriózabal, F. y Gómez A. (30 de abril de 2020) [Telemundo 20]. Esto es lo que sucedió de marzo a abril en Tijuana durante la pandemia del COVID-19. [archivo de video]. Recuperado de https://www.telemundo20.com/noticias/1 ocal/esto-es-lo-que-sucedio-de-marzo-a-abril-en-tiju ana-y-baja-california-durante-la-pandemia-del-covid1 9/2000211/

Casanova, H. (2020). Presentación. En H. Casanova (Coord.), Educación y pandemia. Una visión académica (pp.10-17). México: Universidad Nacional Autónoma de México. Recuperado de http://www.iisue.unam.iisue /covid/educacion-y-pandemia

Delgado, J. Gutiérrez, J. (2007). Métodos y técnicas cualitativas de investigación en ciencias sociales. Madrid, España: Síntesis Psicología.

Durkheim, É. (2006). Sociología y filosofía. Granada, España: Comares.
El Universal [El Universal]. (13 de abril de 2020). Médicos . están cayendo como moscas"por falta de insumos: gobernador de BC [Archivo de video]. Recuperado de https://www.youtube.com/watch?v=61X1Z-uT5WE

Gobierno del Estado de Baja California (31 de marzo de 2020). Vamos a ganar la guerra al COVID-19 en BC con el apoyo de la sociedad, asegura Jaime Bonilla Valdez [Comunicado de prensa] Recuperado de http://www.ba jacalifornia.gob.mx/Gobierno/prensa

Gobierno del Estado de Baja California (17 de marzo de 2020). Confirman en BC 2 casos de coronavirus el Sector Salud hace llamado a la prevención y no al pánico [Comunicado de prensa] Recuperado de http: //www.bajacalifornia.gob.mx/Gobierno/prensa

Gobierno del Estado de Baja California (10 de abril de 2020). Firme la postura del "gobierno en marcha"de no apoyar a las empresas que se niegan a vender respiradores para BC [Comunicado de prensa] Recuperado de http://www. bajacalifornia.gob.mx/Gobierno/prensa

Gobierno del Estado de Baja California (30 de mayo de 2020). Baja California cuenta con infraestructura hospitalaria para la atención de pacientes COVID-19 [Comunicado de prensa] Recuperado de http://www.bajacalifornia.g ob.mx/Gobierno/prensa

Gómez, A. [Telemundo20] (12 de abril de 2020). Eugenio Derbez pide ayuda para hospital en Tijuana. [archivo de video]. Recuperado de https://www.telemundo20.com/ noticias/local/eugenio-derbez-pide-ayuda-para-hospit al-en-tijuana/2009491/

Han, B. (2020). La emergencia viral y el mundo de mañana. En: P. Amadeo (Ed). Sopa de Wuhan: Pensamiento contemporáneo en tiempo de pandemias(pp. 97-112). Aislamiento Social Preventivo y Obligatorio. https: //lareverde.files.wordpress.com/2020/04/sopa-de -wuhan-aspo.pdf

Hermida, A. (2011). Mechanisms of Participation How audience options shape the conversation. En: J. Singer, A. Hermida, D. Domingo, A. Heinonen, S. Paulussen, T. Quandt, Z. Reich, M. Vujnovic (Eds.), Participatory Journalism: Guarding Open Gates at Online Newspapers (pp. 13-33). EUA. Blackwell Publishing Ltd.

Integralia (2019). Planeación, Programación y Rendición de Cuentas del Presupuesto de las Universidades Públicas en México: Razones, Resultados, Retos. México: Integralia. Recuperado de https://integralia.com.m x/web/index.php/2019/08/11/crisis-financiera-de-las-u niversidades-publicas-estatales/

Jodelet, D. (1984). La representación social: fenómenos, conceptos y teoría. En S. Moscovici (comp.), Psicología social (pp. 469-494). Barcelona, España: Paidós.

Jodelet, D. (1997). Les représentations sociales. París, Francia: Presses Universitaires de France. 
Krippendorf, K. (2018). Content analysis: An introduction to its methodology. Los Angeles, EEUU: Sage.

Ksiazek, T. (2018). Commenting on the news, Explaining the degree and quality of user comments on news websites. Journalism Studies, 19(5), 650-673. doi: http://dx.doi.o $\mathrm{rg} / 10.1080 / 1461670 X .2016 .1209977$

López, A. (27 de febrero de 2020). Versión estenográfica de la conferencia de prensa matutina del presidente Andrés Manuel López Obrador. México: AMLO. Recuperado de https://lopezobrador.org.mx/2020/02/27/version-est enografica-de-la-conferencia-de-prensa-matutina-del -presidente-andres-manuel-lopez-obrador-265/

López, A. (28 de febrero de 2020). Versión estenográfica de la conferencia de prensa matutina del presidente Andrés Manuel López Obrador. México: AMLO. Recuperado de https://lopezobrador.org.mx/2020/02/28/version-est enografica-de-la-conferencia-de-prensa-matutina-del -presidente-andres-manuel-lopez-obrador-266/

Markus, G. (2020). El virus, el sistema letal y algunos virus. En: P. Amadeo (Ed.), Sopa de Wuhan: Pensamiento contemporáneo en tiempo de pandemias (pp. 129-134). Aislamiento Social Preventivo y Obligatorio. https: //lareverde.files.wordpress.com/2020/04/sopa-de -wuhan-aspo.pdf

Martí-Noguera J., Calderón, A. y Fernández-Godenzi A. (2018). La responsabilidad social universitaria en Iberoamérica. Revista Iberoamericana de Educación Superior, 24(9) 107-124. Recuperado de https://ries.uni versia.unam.mx/

Martín, M. (2017). La producción social de comunicación. Madrid, España: Alianza.

Martín, M. (2019). La comunicación y la información en un mundo que se virtualiza. Desarrollos y funciones previsibles. Comunicación y Sociedad, 16, 1-29. https: //doi.org/10.32870/cys.v2019i0.7478

Martín, M. y Velarde O. (2015). La mediación comunicativa de las identidades individuales y colectivas. Revista Latina de Comunicación Social, 70, 552-565. doi: 10.4185/RLCS-2015-1059

McCombs, M. (1996). Influencia de las noticias sobre nuestras imágenes del mundo. En B. Jennings D. Zillmann (comps.). Los efectos de los medios de comunicación: Investigaciones y teorías (pp.13-34). Barcelona, España: Paidós Comunicación.

Moscovici, S. (1979). El psicoanálisis, su imagen y su público. Buenos Aires, Argentina: Editorial Huemul.

Moscovici, S. (1988). Notes towards a definition of social representations. European Journal of Social Psychology, 18, 211-250.

Neuendorf, K. (2017). The Content Analysis Guidebook. Los Angeles, EEUU: Sage.

Organización Mundial de la Salud (27 de abril de 2020). COVID-19: cronología de la actuación de la OMS. OMS. Recuperado de www.who.int.
Organización Mundial de la Salud (2020). Preguntas $y$ respuestas sobre la enfermedad por coronavirus (COVID-19). OMS. Recuperado de www.who.int

Piñera D. (2008). Las universidades de los estadosde la república en el contexto nacional.En M. Alvarado y L. Pérez (Coords.) Cátedras y catedráticos en la historia de las universidades e instituciones de educación superior en México.III. Problemática universitaria en el siglo XX (pp. 71-82). México: IISUE-UNAM.

Piñuel, J. (2002). Epistemología, metodología y técnicas del análisis del contenido. Estudios de sociolingüística, 3(1), 1-42. http://www.sociolinguis-tica.uvigo.es/articulosXv olumen.asp?id=10

Reyes, K. [El Imparcial Mexicali] (12 de abril de 2020). Faltan médicos y ventiladores para atender el COVID-19 en BC. [Archivo de video]. Recuperado de https://www.el imparcial.com/mexicali/mexicali/Faltan-medicos-y-v entiladores-para-atender-el-Covid-19-en-BC-2020041 2-0001.html

Rosagel, Shaila [El Imparcial Tijuana] (5 de abril de 2020). Llama AMLO a más médicos para luchar vs. COVID-19. Recuperado de https://www.elimparcial.com/tijuana/tiju ana/Llama-AMLO-a-mas-medicos-para-luchar-contr a-Covid-19-20200405-0001.html

Secretaría de Educación Pública (SEP) (2020). Acuerdo 02/03/2020. México: SEP. Recuperado de https://www. dof.gob.mx/nota_detalle.php?codigo $=5589479 \&$ fecha $=16 / 03 / 2020$

Secretaría de Salud del Gobierno de México (SSGM) (28 de febrero de 2020). Comunicado Técnico Diario Nuevo Coronavirus en el Mundo (COVID-19) [Comunicado de prensa]. Recuperado de https://www.gob.mx/cms/uploa ds/attachment/file/538453/Comunicado_Tecnico_Diari o_COVID-19_2020.02.28.pdf

Secretaría de Salud del Gobierno de México (SSGM) (24 de marzo de 2020). Inicia fase 2 por coronavirus de COVID-19. [Comunicado de prensa]. Recuperado de https:/coronavirus.gob.mx/2020/03/24/inicia-fase-2por-coronavirus-covid-19/

Secretaría de Salud del Gobierno de México (SSGM) (30 de mayo de 2020). Información del nuevo coronavirus [Comunicado de prensa]. Recuperado de https://www.go b.mx/salud/prensa/nuevo-coronavirus-en-el-mundo-co vid-19-comunicado-tecnico-diario-243699?idiom=es

Segado-Boj, F., Díaz-Campo, J. y Navarro-Sierra, N. (2020). Emociones y difusión de noticias sobre el cambio climático en redes sociales. Influencia de hábitos, actitudes previas y usos y gratificaciones en universitarios. Revista Latina de Comunicación Social, 75, 245-269. https://www.doi.org/10.4185/RLCS-20201425

Tausczik, Y. y Pennebaker, J. (2009). The Psychological Meaning of Words: LIWC and Computerized Text Analysis Methods. Journal of Language and Social 
Psychology, 29(24), 24-54. http://jls.sagepub.com/cont ent/29/1/24.

Taylor, S. y Bogdan, R. (1987). Introducción a los métodos cualitativos de investigación. Barcelona, España: Paidós Básica.

Tecnológico de Monterrey (12 de marzo de 2020). Comunicado Coronavirus - Tecnológico de Monterrey. México: Tec de Monterrey. Recuperado de: https://tec. $\mathrm{mx} /$ sites/default/files/inline-files/Comunicado-OficialCovid-Mzo-12-tec.pdf

Universidad Autónoma de Baja California (UABC) (2020). Sistema Institucional de Indicadores. México: UABC. Recuperado de: http://www.uabc.mx/planeacion/numer alia/

Universidad Autónoma de Baja California (UABC) (2019). Plan de Desarrollo Institucional 2019-2023. Recuperado de: http://www.uabc.mx/planeacion/pdi/2019-2023/P DI_2019-2023.pdf

Universidad Nacional Autónoma de México (UNAM) (12 de marzo de 2020). Boletín UNAM-DGCS-224. México: UNAM. Recuperado de: https://www.dgcs.unam.mx/bol etin/bdboletin/2020_224.html

Vallaeys, F. (2018). Las diez falacias de la Responsabilidad Social Universitaria. Revista Digital de Investigación en Docencia Universitaria, 12(1), 34-58. doi: http://dx.doi .org/10.19083/ridu.12.716

Vallaeys, F. (2006). Breve marco teórico de la responsabilidad social universitaria. Lima: Banco Interamericano de Desarrollo.

Velarde, O. y Bernete, F. (2016). La producción social de comunicación cuando el mundo se globaliza. JANUS.NET e-journal of International Relations, 7(2), 90-103. Recuperado de https://repositorio.ual.pt/bitstre am/11144/2785/3/en_vol7_n2_art6.pdf

Villalobos, S. [El Financiero] (13 de abril de 2020). Falta de materiales en hospital del IMSS de Baja California es real, no fake news: Eugenio Derbez. [archivo de video]. Recuperado de https://www.elfinanciero.com.mx/tv/el-f inanciero-bloomberg-20-hrs-con-sofia-villalobos/falt a-de-materiales-en-hospital-del-imss-de-baja-califor nia-es-real-no-fake-news-eugenio-derbez

Wahl-Jorgensen, K. (2019). An Emotional Turn in Journalism Studies?. Digital Journalism, 8(2), 175-194. doi: https: //doi.org/10.1080/21670811.2019.1697626

Zizek, S. (2020). Pandemia: La covid-19 estremece al mundo. España: Anagrama. 